\title{
Structural reliability analysis of a planar truss in case of limited statistical data
}

\author{
Sergey A. Solovyev ${ }^{1, *}$, and Anastasia A. Solovyeva ${ }^{1}$ \\ ${ }^{1}$ Vologda State University, Industrial and Civil Construction Department, Russian Federation
}

\begin{abstract}
Trusses are common structural elements in many industrial and civil buildings. The article describes the approach to planar trusses reliability analysis in case of limited statistical data. The proposed approach can be used when it is complicated to determine a cumulative distribution function for design loads and physical/mechanical properties of the material of structural elements. The proposed method for structural reliability analysis requires only estimates of mathematical expectations and standard deviations. The article also presents the equation for planar truss reliability assessment as a system of structural elements. The result of a planar truss reliability analysis is obtained at an interval of non-failure probabilities. If the resulting reliability interval is too wide for decision-making, it is necessary to improve the quality of statistical data for a more accurate assessment of reliability (non-failure probability).
\end{abstract}

\section{Introduction}

Planar trusses are common structural elements in many industrial and civil buildings [1-5]. The issues of reliability are of high priority in various industries [6$10]$ and it is highly relevant for construction industry, so development and modernization of approaches to trusses safety assessment is an actual scientific problem because there are some cases of trusses failure caused by errors of structural engineers in recent years $[11,12]$.

As noted in [13], the analysis and design of structures based on reliability theory is a topic that has recently been seriously considered. This attention is associated with the random nature of the structural parameters, such as material properties, external loads, geometric characteristics of the cross-section of members, geometric dimensions of structures, and so on. Using reliability theory in structural systems, the uncertainties caused by the statistical nature of the structural parameters can be introduced as mathematical equations, while the safety and performance considerations are applied quantitatively in the design process [13].

The article [13] presents the approach to structural reliability analysis of truss structures with natural frequency constraints using metaheuristic algorithms. The paper [14] describes the reliability-based evaluation for concrete-filled steel tubular (CFST) truss under flexural loading. Reliability analysis is conducted to calculate reliability indexes in respect to different resistance factors under various load cases.

This article presents the probabilistic approach to structural reliability analysis of planar trusses. The "Molodechno" type truss (Fig.1) will be considered as an example.

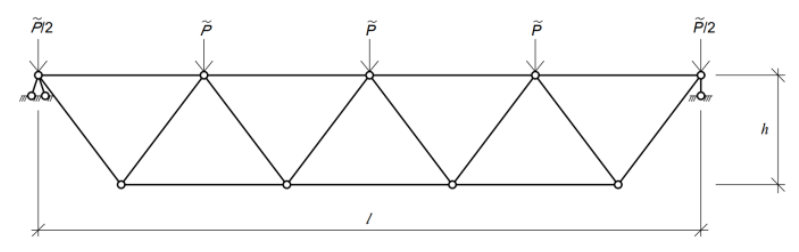

Fig. 1. Example of "Molodechno" planar truss with a random (stochastic) load.

\section{Methods}

The stress analysis of a planar truss can be performed using classical approaches, such as the moment point method (Fig. 2).

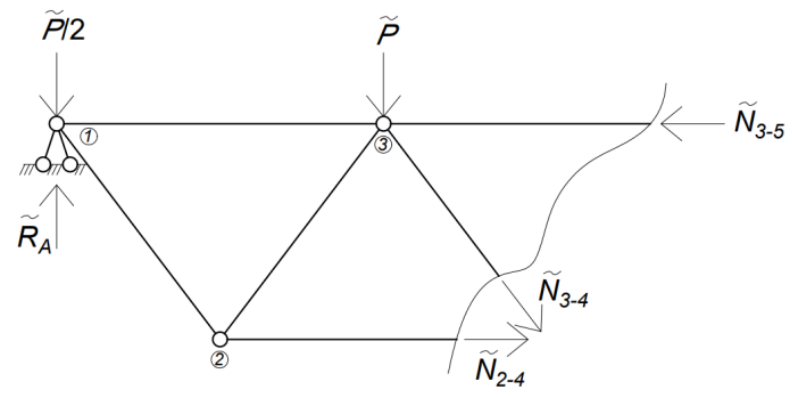

Fig. 2. The example of the moment point method in a planar truss structural design.

The force $\tilde{N}_{3-5}$ can be found from the condition:

$$
\begin{gathered}
\sum M_{4}=0 \rightarrow N_{3-5}^{\circ} \cdot h+\beta / \beta_{0} 0.5 \cdot l_{2-4}+ \\
+0.5 \cdot \beta \circ\left(0.5 \cdot l_{1-3}+l_{2-4}\right)-R_{A}^{\circ} \cdot\left(0.5 \cdot l_{1-3}+l_{2-4}\right)=0
\end{gathered}
$$

\footnotetext{
* Corresponding author: solovevsa@,vogu35.ru
} 
where $\tilde{N}_{i-j}$ is an internal force in the $i-j$ truss bar; $h$ is a truss height; $l_{i-j}$ is a length of the $i-j$ truss bar.

From eq. (1) it can be seen that an each force $\tilde{N}_{i-j}$ can be represented as:

$$
\widetilde{N}_{i-j}(\widetilde{P})=\widetilde{P} \cdot \alpha
$$

where $\alpha$ is a geometrical coefficient of a truss shape.

The limit state condition for every structural element in a planar truss can be presented as:

$$
\tilde{N}_{i-j}(\widetilde{P}) \leq \widetilde{N}_{i-j, u l t},
$$

where $\tilde{N}_{i-j, u l t}$ is an ultimate force for $i-j$ structural element in a planar truss.

An ultimate force for $i-j$ structural element can be determined by different limit state criteria.

For example, for the truss bar strength criteria:

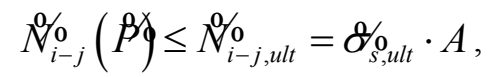

where $A$ is a bar cross-section area; $\widetilde{\sigma}_{S, u l t}$ is an ultimate stress (the random variable).

For a bar buckling criteria:

$$
N_{i-j}^{0}(\beta) \leq N_{i-j, u l t}^{0}=\sigma_{s, u l t}^{0} \cdot A \cdot \phi,
$$

where $\varphi$ is a buckling factor.

A random variable is characterized by a distribution function. Probabilistic cumulative distribution functions are used in the classic structural reliability theory. In case of limited statistical information, boundary probability functions are used. Such functions are called probability box or p-box [15].

The papers $[15,16]$ define the bounds of a free p-box by the two-sided Chebyshev's inequality [17]:

$$
\begin{gathered}
\underline{F}_{X}(x)=\left\{\begin{array}{c}
0, \text { for } x<m_{X}+S_{X} \\
1-\frac{S_{X}^{2}}{\left(x-m_{X}\right)^{2}}, \text { for } x \geq m_{X}+S_{X},
\end{array}\right. \\
\bar{F}_{X}(x)=\left\{\begin{array}{cc}
\frac{S_{X}^{2}}{\left(x-m_{X}\right)^{2}}, \text { for } x<m_{X}-S_{X}, \\
1, \text { for } x \geq m_{X}-S_{X}
\end{array}\right.
\end{gathered}
$$

where $m_{X}$ is an expected value (a mathematical expectation); $S_{X}$ is an standard deviation.

The advantage of this probability box is an applicability in the presence of statistical estimates of the expected value $m_{X}$ and standard deviation $S_{X}$. In this case there is no need to test the statistical hypothesis about the distribution function (Pearson's chi-squared test, for example). However, the result of the reliability analysis will be presented as an interval, not as a discrete value.
Tighter bounds can be obtained by using one-sided Chebyshev's inequalities and knowledge about the minimum $\underline{x}$ / maximum $\bar{x}$ value of $X$ [16]:

$$
\begin{aligned}
& 0, \text { for } x \leq m_{X}+S_{X}^{2} /\left(m_{X}-\bar{x}\right) \\
& \bar{F}_{X}(x)=\left\{\begin{array}{c}
1-\left[b(1+a)-c-b^{2}\right] / a, \\
\text { for } m_{X}+S_{X}^{2} /\left(m_{X}-\bar{x}\right)<x<m_{X}+S_{X}^{2} /\left(m_{X}-\underline{x}\right) \\
1 /\left[1+S_{X}^{2} /\left(x-m_{X}\right)^{2}\right], \text { for } m_{X}+S_{X}^{2} /\left(m_{X}-\underline{x}\right)<x<\bar{x} \\
1, \text { for } x \geq \bar{x}
\end{array},\right. \\
& \underline{F}_{X}(x)=\left\{\begin{array}{c}
0, \text { for } x \leq \underline{x} \\
1 /\left[1+\left(x-m_{X}\right)^{2} / S_{X}^{2}\right], \text { for } \underline{x}<x<m_{X}+S_{X}^{2} /\left(m_{X}-\bar{x}\right),(6) \\
1-\left(b^{2}-a b+c\right) /(1-a), \\
\text { for } m_{X}+S_{X}^{2} /\left(m_{X}-\bar{x}\right)<x<m_{X}+S_{X}^{2} /\left(m_{X}-\underline{x}\right) \\
1, \text { for } x \geq m_{X}+S_{X}^{2} /\left(m_{X}-\underline{x}\right)
\end{array}\right.
\end{aligned}
$$

where $\quad x \in\lfloor\underline{x}, \bar{x}\rfloor ; \quad$ parameters: $\quad a=(x-\underline{x}) /(\bar{x}-\underline{x})$, $b=\left(m_{X}-\underline{x}\right) /(\bar{x}-\underline{x})$ and $c=S_{X}^{2} /(\bar{x}-\underline{x})^{2}$.

\section{Results}

The functions (3) and (4) form p-boxes that cover the true probability distribution function (PDF) of a random variable. For example, assume that the following statistical estimates were obtained for the force in the truss bar: $m_{X}=20 \mathrm{kN}, S_{X}=1.5 \mathrm{kN}$. Then the $p$-boxes by (3)-(4) and (5)-(6) equations form an epistemic uncertainty area (Fig. 1).

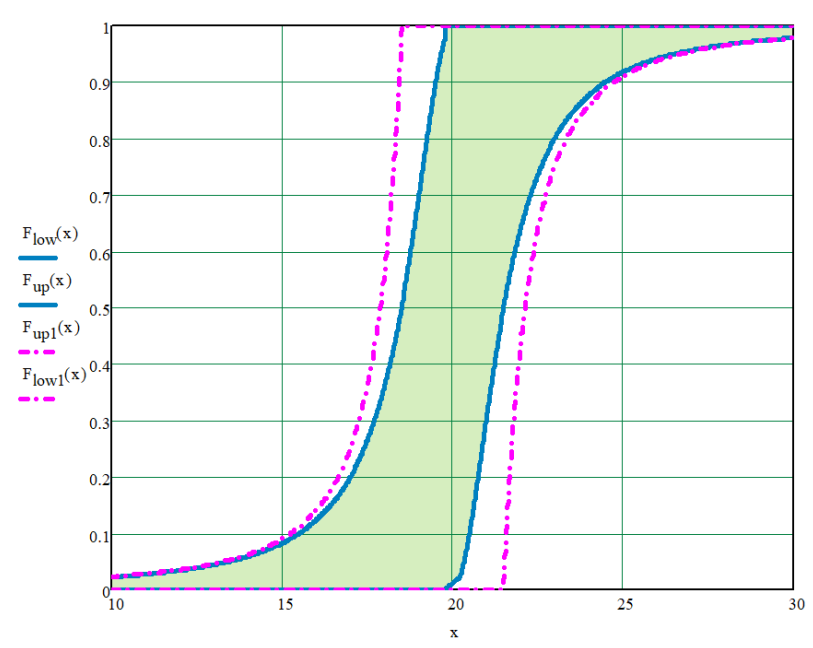

Fig. 3. The PDF area formed by p-box (5)-(6).

As can be seen from Fig. 3, the probability distribution functions (5)-(6) form the tighter p-box. So, the equations (5)-(6) can be used for the random variables $\tilde{N}_{i-j}(\widetilde{P})$ and $\tilde{\sigma}_{s, u l t}$.

The limit state condition (2) can be presented as:

$$
X \leq Y .
$$


Boundaries of failure probability $Q$ can be estimated for boundary distribution functions using the following equations:

$$
\begin{aligned}
& \underline{Q}=\int_{0}^{\infty} \bar{f}_{X}(x) \cdot \underline{F}_{Y}(x) d x, \\
& \bar{Q}=\int_{0}^{\infty} \underline{f}_{X}(x) \cdot \bar{F}_{Y}(x) d x,
\end{aligned}
$$

where $\bar{f}_{X}(x)=\frac{d \bar{F} X(x)}{d x}$ is the probability density function for $\bar{F} X(x)$.

The non-failure probabilities can be calculated as $\lfloor\underline{P}=1-\bar{Q}, \bar{P}=1-\underline{Q}\rfloor$.

The table with reliability intervals for truss bars can be formed for a plane truss. Table 1 is an example of such table.

Table 1. Reliability of planar truss elements.

\begin{tabular}{|c|c|c|}
\hline $\begin{array}{c}\text { Planar truss element } \\
\text { (Fig. 1) }\end{array}$ & $\underline{P}$ & $\bar{P}$ \\
\hline $1-2$ & 0.9942 & 0.9989 \\
\hline $1-3$ & 0.9992 & 0.9999 \\
\hline $2-3$ & 0.9942 & 0.9989 \\
\hline $2-4$ & 0.9967 & 0.9993 \\
\hline $3-4$ & 0.9975 & 0.9995 \\
\hline $3-5$ & 0.9970 & 0.9990 \\
\hline $4-5$ & 0.9975 & 0.9995 \\
\hline $4-6$ & 0.9953 & 0.9988 \\
\hline $5-6$ & 0.9975 & 0.9995 \\
\hline $5-7$ & 0.9970 & 0.9990 \\
\hline $6-7$ & 0.9975 & 0.9995 \\
\hline $6-8$ & 0.9967 & 0.9993 \\
\hline $7-8$ & 0.9942 & 0.9989 \\
\hline $7-9$ & 0.9992 & 0.9999 \\
\hline $8-9$ & 0.9942 & 0.9989 \\
\hline
\end{tabular}

\section{Discussion}

The planar truss reliability can be represented as a sequential system in terms of the reliability theory. In classic reliability theory, the next equation is used for reliability assessment of a sequential system: $\prod_{i=1}^{n} P_{i}$, where $P_{i}$ is a non-failure probability of $i$-th element in system. In case of limited statistical data, a result of the structural reliability analysis is presented in the interval form, as noted above. Then, the truss reliability assessment as a mechanical system can be provided using the following equation:

$$
\left\{\begin{array}{c}
\underline{P}=\max \left(0, \sum_{i=1}^{n} \underline{P}_{i}-(n-1)\right), \\
\bar{P}=\min \left(\bar{P}_{i}\right)
\end{array}\right.
$$

where $\underline{P}_{i}$ and $\bar{P}_{i}$ are lower and upper bounds of pile non-failure probability by $i$-th element of a truss.

The advantage of equations (10) is also the ability to use them in the absence of statistical data on the dependence (independence) of elements in the system.

For statistical data in Table 1, the following bounds can be obtained:

$$
\begin{gathered}
\underline{P}=\max \left(0, \sum_{i=1}^{n} \underline{P}_{i}-(n-1)\right)=14.9479-(15-1)=0.9479, \\
\bar{P}=\min \left(\bar{P}_{i}\right)=0.9988 .
\end{gathered}
$$

The reliability of planar truss is [0.9479; 0.9988]. If this interval is too wide to make a decision about the level of truss safety, then the quality of statistical information should be improved: probability functions of distributions should be refined, the number of control samples should be increased, etc.

\section{Conclusion}

1. The article describes the approach to a planar truss reliability analysis in case of limited statistical data about design loads and mechanical properties of truss material.

2 . The result of a planar truss reliability analysis is an interval of non-failure probabilities. If the resulting reliability interval is too wide for decision-making, it is necessary to improve the quality of statistical data for a more accurate assessment of reliability (non-failure probability).

3. The numerical example is given for the planar truss reliability analysis as a mechanical system.

\section{References}

1. N. Wicks and J. W. Hutchinson, Int. J. Solids Struct. (2001)

2. B. De Jager and R. E. Skelton, Int. J. Solids Struct. (2006)

3. A. Y. N. Sofla, D. M. Elzey, and H. N. G. Wadley, Smart Mater. Struct. (2009)

4. A. Kaveh and A. Zolghadr, Adv. Eng. Softw. (2015)

5. G. G. Tejani, V. J. Savsani, V. K. Patel, and P. V. Savsani, J. Comput. Des. Eng. (2018)

6. I. G. Akhmetova, A. A. Kalyutik, A. V Fedukhin, O. V Derevianko, and L. R. Mukhametova, in (2019)

7. A. Udaratin, A. Alyunov, A. Krutikov, L. R. Mukhametova, O. O. Zaripov, and I. V. Bochkarev, E3S Web Conf. 124, 02020 (2019)

8. I. Kadyrov, N. Karaeva, Z. Andarbekov, B. Azamat, O. Fedorov, and O. Vladimirov, E3S Web Conf. 178, 01036 (2020)

9. S. T. Latipov, G. N. Aslanova, L. A. Nematov, A. A. Akhmedov, and M. R. Charieva, E3S Web Conf. 139, (2019) 
10. D. Jato-Espino, E. Castillo-Lopez, J. RodriguezHernandez, and J. C. Canteras-Jordana, Autom. Constr. (2014)

11. R. M. Latture, M. R. Begley, and F. W. Zok, J. Mater. Res. (2018)

12. R. S. Nagavi and A. E. Aktan, J. Struct. Eng. (2003)

13. S. R. Hoseini Vaez, H. Mehanpour, and M. A. Fathali, J. Build. Eng. 28, 101065 (2020)

14. S. Chen, C. Hou, H. Zhang, L. H. Han, and T. M. $\mathrm{Mu}$, J. Constr. Steel Res. 169, 106018 (2020)

15. M. Oberguggenberger and W. Fellin, Comput. Struct. 86, 1093 (2008)

16. H. Zhang, H. Dai, M. Beer, and W. Wang, Mech. Syst. Signal Process. 37, 137 (2013)

17. C. L. Mallows, J. R. Stat. Soc. Ser. B 18, 139 (1956)

18. L. V. Utkin and S. V. Gurov, Knowl. Inf. Syst. 1, 459 (1999). 